\title{
The relation between graphing formulas by hand and students' symbol sense
}

\author{
Peter M.G.M. Kop ${ }^{1}$ • Fred J.J.M. Janssen ${ }^{1}$ • Paul H.M. Drijvers ${ }^{2}$ • Jan H. van Driel ${ }^{3}$ \\ Published online: 24 September 2020 \\ (C) The Author(s) 2020
}

\begin{abstract}
Students in secondary school often struggle with symbol sense, that is, the general ability to deal with symbols and to recognize the structure of algebraic formulas. Fostering symbol sense is an educational challenge. In graphing formulas by hand, defined as graphing using recognition and reasoning without technology, many aspects of symbol sense come to play. In a previous study, we showed how graphing formulas by hand could be learned. The aim of the study we present here is to explore the relationship between students' graphing abilities and their symbol sense abilities while solving nonroutine algebra tasks. A symbol sense test was administered to a group of 114 grade 12 students. The test consisted of eight graphing tasks and twelve non-routine algebra tasks, which could be solved by graphing and reasoning. Six students were asked to think aloud during the test. The findings show a strong positive correlation between the scores on the graphing tasks and the scores on the algebra tasks and the symbol sense used while solving these tasks. The thinking-aloud protocols suggest that the students who scored high on the graphing tasks used similar aspects of symbol sense in both the graphing and algebra tasks, that is, using combinations of recognizing function families and key features, and qualitative reasoning. As an implication for teaching practice, learning to graph formulas by hand might be an approach to promote students' symbol sense.
\end{abstract}

Keywords Graphing formulas · Qualitative reasoning · Symbol sense

Peter M.G.M. Kop

koppmgm@iclon.leidenuniv.nl

1 ICLON Leiden University Graduate School of Teaching, Postbox 905, 2300 AX Leiden, the Netherlands

2 Utrecht University, Princetonplein 5, 3584 CC Utrecht, the Netherlands

3 The University of Melbourne, Parkville 3010 VIC, Australia 


\section{Introduction}

Many students have serious cognitive problems with algebra, in particular with seeing structure and making sense of algebraic formulas with their abstract symbols (Arcavi, Drijvers, \& Stacey, 2017; Drijvers, Goddijn, \& Kindt, 2011; Kieran, 2006). The teaching of algebra often focuses on basic skills through practicing algebraic calculation in similar tasks (Arcavi et al., 2017). However, many students experience problems with when to use these basic skills and finding strategies to solve algebra problems: they lack symbol sense (Arcavi et al., 2017; Hoch \& Dreyfus, 2005, 2010; Oehrtman, Carlson, \& Thompson, 2008; Thompson, 2013). Symbol sense concerns a very general notion of "when and how" to use symbols (Arcavi, 1994), and it functions as a compass when using basic skills (Drijvers et al., 2011). A lack of symbol sense leads to an overreliance on basic skills, just learned methods, and on the symbolic representations, leading to poor achievements (Kieran, 2006; Knuth, 2000; Eisenberg \& Dreyfus, 1994; Pierce \& Stacey, 2007). However, it is not clear how to teach symbol sense appropriately (Arcavi, 2005; Hoch \& Dreyfus, 2005). In a previous study, we showed how teaching graphing formulas by hand, defined as graphing using recognition and reasoning, without technology, to grade 11 students improved their insight into algebraic formulas (Kop, Janssen, Drijvers, \& Van Driel, 2020). Insight into algebraic formulas is an aspect of symbol sense and involves recognizing structure and key features of a formula and qualitative reasoning with and about a formula. In the study presented here, we investigated whether graphing formulas by hand abilities is related to students' symbol sense in a broader sense, that is, symbol sense while solving non-routine algebra tasks. Doing so, the study aims to contribute to our theoretical knowledge of students' symbol sense abilities and to inform teaching practice.

\section{Theoretical background}

The most important theoretical notion that guides this study is symbol sense. Fey (1990) was the first to mention symbol sense and described it as an informal skill required to deal effectively with symbolic expressions and algebraic operations. According to Fey, goals for teaching symbol sense would include at least the following basic themes: the ability to scan an algebraic expression to make rough estimates of the patterns that would emerge in numerical or graphical representation, to make comparisons of orders of magnitude for functions, and to inspect algebraic operations and predict the form of the result and judge the likelihood that it has been performed correctly. Arcavi (1994) elaborated on this idea and broadened the concept to all phases in the problem-solving cycle (Pierce \& Stacey, 2004). According to Arcavi (ibid.), symbol sense would include:

- An understanding and a feel for the power of symbols, that is, understanding how and when symbols can be used to display relationships, generalizations, and proofs and when to abandon symbols in favor of other approaches in order to make progress with a problem

- An ability to manipulate and to "read" symbolic expressions as two complementary aspects of solving algebra problems

- The awareness that one can successfully engineer symbolic relationships which express the verbal or graphical information needed to make progress in a problem, and the ability to engineer those expressions 
Drijvers et al. (2011) described symbol sense in relation to basic skills: symbol sense and basic skills are complementary. Basic skills involve procedural work with a local focus and an emphasis on algebraic calculations, whereas symbol sense involves strategic work, taking a global view on algebraic expressions/formulas and algebraic reasoning. A global view, or a Gestalt view, has to do with the ability to see an algebraic formula as a whole, to "read through" it, and to recognize its structure and global characteristics.

Related to the notion of symbol sense, Pierce and Stacey (2004) used the term algebraic insight to capture the symbol sense in transformational activities in the "solving" phase of the problem-solving cycle (from mathematical problem to mathematical solution) when using Computer Algebra Systems (CAS). Algebraic insight has to do with the recognition and identification of structure, objects, key features, dominant terms, and meanings of symbols and the ability to link representations (Kenney, 2008; Pierce \& Stacey, 2004). Kenney (2008) used this framework of Pierce and Stacey for her research and added "know how and when to use symbols" and "know when to abandon a representation."

To develop symbol sense, graphing formulas by hand might be useful because it involves many aspects of symbol sense. First, graphing formulas is about linking the symbolic and graphical representation. Second, to efficiently graph formulas by hand, one has to recognize the structure and key features of a formula and to reason with and about formulas. Third, graphing a formula can be considered a visualization of a formula, and using such a visualization in problem solving requires knowing about what is represented and where to look for it. We will now elaborate on these aspects.

Linking representations, such as formulas and graphs, is important in learning about functions (Janvier, 1987; Leinhardt, Zavlavsky, \& Stein, 1990) and might be used to give meaning to algebraic formulas (Kieran, 2006). Duval (1999) used the term registers of representations to indicate that each representation (formula or graph) has its own specific means and processing for mathematical thinking. He distinguished two types of transformation: treatments, transformations in the same representation, and conversions, transformations from one representation to another, like from formulas into Cartesian graphs. Conversions are at the core of understanding mathematics, but many students have problems learning these conversions, as it requires a change of register and the recognition of the same represented object in different representations (Duval, 1999, 2006).

In efficiently graphing formulas by hand, many aspects of symbol sense are involved. Research in expertise in graphing formulas by hand shows that experts' strategies could be described with combinations of different levels of recognition and qualitative reasoning (Kop, Janssen, Drijvers, Veenman, \& Van Driel, 2015). For recognition, experts use a repertoire of function families with their characteristics and key graph features like zeroes and turning points. In qualitative reasoning, the focus is on the global shape of the graph, ignoring what is not relevant in the situation, and using global descriptions. Qualitative reasoning is often used by experts in complex problem situations that are difficult to look through in detail, like in physical models (Bredeweg \& Forbus, 2003). In the domain of graphing formulas, experts tend to use qualitative reasoning to explore (parts of) the graph, for instance, infinity behavior, increasing/decreasing of functions, stronger/weaker components of a function, and in the composition of two subgraphs, after decomposing a formula in two sub-functions. Graphing formulas by hand is related to covariational reasoning, which is about coordinating two covarying quantities while attending to how they change in relation to each other (Thompson, 2013; Carlson, Jacobs, Coe, Larsen, \& Hsu, 2002). This covariational reasoning is critical in supporting student learning of functions in secondary and undergraduate 
mathematics (Carlson et al., 2002; Oehrtman et al., 2008). Carlson, Madison, and West (2015) showed that students were not able to reason "as the value of $x$ gets larger the value of $y$ decreases, and as the value of $x$ approaches 2, the value of $y$ increases" when they had to link the formula of $f(x)=1 /(x-2)^{2}$ to its graph.

Visualizing formulas through graphs is used in problem solving for understanding the problem situation, recording information, exploring, and monitoring and evaluating results (Polya, 1945; Stylianou \& Silver, 2004). Stylianou and Silver (2004) compared experts and novices in solving algebra problems and showed that experts know how to use graphs in solving algebra problems. Experts "see" relevant relations visualized in the graph and can use the graph for visual and qualitative explorations. Although novices have some declarative knowledge, they lack the necessary procedural knowledge to construct visual representations of general functions and to explore the graphs they have constructed. Such exploration requires a global view of the whole graph and not just a local apprehension (Duval, 1999) and is only possible when one is very familiar with the function (Stylianou \& Silver, 2004). This matched Eisenberg and Dreyfus' (1994) ideas about the need for a repertoire of basic functions that one should simultaneously "see" in a graph as one thinks of the algebraic formula.

In sum, graphing formulas involves many essential aspects of symbol sense to solve algebra problems, like visualizing a formula through a Cartesian graph, taking a global view to read through a formula and enable recognition of the structure of a formula and/or its key features, and qualitative reasoning. In the current study, therefore, we focus on the relation between symbol sense involved in graphing formulas by hand and the symbol sense to solve non-routine algebra tasks. Aspects of symbol sense, learned and used in the context of graphing formulas, might be used in a broader domain of algebra tasks. In this study, this broader domain is restricted to algebra tasks that can be solved with graphs and reasoning, so without the use of algebraic calculations.

\subsection{Research questions}

The theoretical perspective described in the previous section led to the following main research question:

How do grade 12 students' abilities to graph formulas by hand relate to their use of symbol sense while solving non-routine algebra tasks?

We formulated two sub-questions. We expected a relation between students' abilities to graph formulas by hand and their abilities to solve algebra tasks with symbol sense, because graphing formulas can be seen as a subset of algebra tasks, and in graphing formulas by hand, many aspects of symbol sense are involved. This led to the first sub-question:

To what extent are students' graphing formulas by hand abilities positively correlated to their abilities to solve algebra tasks with symbol sense?

In graphing formulas by hand, several symbol sense aspects are involved, and we expected that students would be able to use these symbol sense aspects also in the context of solving algebra tasks. In addition, we expected that when one is able to graph formulas by hand, one would see more possibilities to use this strategy (making a graph). This led to the second subquestion: 
How is students' symbol sense use in graphing formulas similar or different from their symbol sense use in solving non-routine algebra tasks?

\section{Method}

We first describe the context of the study, that is, the position of this study in a larger research project; next, we address the participants, the symbol sense test, the data, and the way the data were analyzed.

\subsection{Context of the study}

This study is part of a larger $\mathrm{PhD}$ research project about studying how symbol sense might be taught. In two previous studies, we analyzed expertise in graphing formulas by hand and identified main components of symbol sense used by experts, that is, recognition of function families and key features from the structure of formulas and qualitative reasoning. In a third study, a group of 21 students from the first author's school were taught how to graph formulas by hand, using recognition and reasoning, in a series of five lessons of $90 \mathrm{~min}$. The series of lessons started with the recognition of basic function families with their characteristics. Students learned about transformations and about using qualitative reasoning by focusing on the global shape of the graphs and using global descriptions (e.g., "it is a root-graph reversed"). Then, these basic function families were used as building blocks when graphing more complex functions, like in $y=2 x+4 / x$ and $y=2 x \sqrt{5-x}$. Complex functions could be decomposed in two basic functions, which could both be graphed. Explicit attention was paid to the composition of the two subgraphs through qualitative reasoning. In a subsequent task, the focus was on recognizing graph features, like zeroes and turning points. When recognition falls short, one can do strategic explorations of parts of the graphs. In a subsequent task, students learned how to use qualitative reasoning for determining, e.g., infinity behavior of a function and increasing/decreasing. In Appendix 3, more details are given about this intervention. Pre-test results of this third study showed that the students had a lot of trouble with graphing formulas by hand; post-test results showed an improvement of their abilities. The current study is the fourth study, which focused on the relation between symbol sense involved in graphing formulas by hand and in solving algebra tasks.

\subsection{Instruments}

The main instrument developed for this study was a test on students' competencies and symbol sense use when graphing formulas by hand and when solving non-routine algebra tasks. Two types of tasks were used: type A tasks, in which the link between formula and graph was explicitly indicated, and type B tasks with no reference to graphs in the text. Students were asked to explain their answers.

The test was constructed in three steps. First, we looked for tasks that were used in other studies and adjusted them to our situation. Second, a first draft of the test was discussed with a professor in mathematics education and an experienced teacher. They were asked whether the tasks fit the grade 12 curriculum and whether they thought the students should be able to solve these tasks. Third, using their feedback, the test was constructed with eight type A tasks and 
twelve type B tasks. All teachers of the students involved in the study indicated that they thought that these tasks were challenging but, according to the curriculum, should be doable.

In the type A tasks, we explicitly used the word "graph" and addressed different aspects of linking formulas to graphs. Some of these kinds of tasks have been used more often in research: working with parameters (Drijvers et al., 2011; Heid, Thomas, \& Zbiek, 2013), reverse thinking (finding a formula with a graph) (Keller, 1994; Drijvers et al., 2011; Duval, 2006), and evaluating a (part of the) graph made with a graphic calculator. The test started with type B tasks, because type A tasks might suggest using graphs in the type B tasks.

Type B tasks could be solved with only graphs and reasoning, but no explicit links to graphs were given in the text. These tasks should give information about the students' symbol sense use while solving algebra tasks. Some tasks have been used by others in assessing students' algebraic competences: number of solutions (Heid et al., 2013), inequalities (Kenney, 2008; Tsamir \& Bazzini, 2004), and reasoning about the function (Kenney, 2008; Pierce \& Stacey, 2007). In Appendix 1, we give the symbol sense test. The internal consistency and reliability of both types of tasks was deemed acceptable, based on Cronbach's alpha on the type A tasks being 0.70 and on the type B tasks 0.72 . Deleting any task hardly changed Cronbach's alpha.

\subsection{Participants}

In this study, 114 grade 12 students from six different schools throughout the Netherlands were involved. The students had $45 \mathrm{~min}$ to finish the pen-and-paper test. All students were enrolled in the Dutch mathematics B course that prepares for university studies in mathematics, physics, and engineering. In regular education in the Netherlands, students learn about linear, quadratic, and exponential functions in grade 8 and 9. In grade 10, the graphic calculator is introduced, and power, rational, logarithmic functions are studied. In grade 11 and 12, calculus topics such as derivatives and integrals are taught. Graphing formulas by hand, without technology, is not a specific subject in the Dutch curriculum: graphs are normally made with the graphic calculator. Therefore, we expected that many students would have difficulties to graph formulas by hand and that they would score low on the graphing tasks in the symbol sense test. To investigate the relation between graphing formulas abilities and the abilities to solve algebra tasks, a broad range of scores on the graphing tasks was needed. To ensure this range of graphing abilities and to investigate how the teaching of graphing formulas by hand would affect students' symbol sense abilities, 21 students from the third study (who were taught how to graph formulas by hand) were involved in the current study. The teachers of the five schools that were involved in the study volunteered to participate, and differed with respect to years of teaching experience.

\subsection{Procedure}

In February 2017, the symbol sense test was administered to the 114 students. For each student, all answers on the tasks were scored as correct (score $=1$ ), partly correct (score between 0 and 1), or incorrect (score $=0$ ). For each student, the sum of the scores on the type A tasks resulted in a TA score and the sum on type B tasks in a TB score. In addition, for both type A and type B tasks, the students' strategies were encoded, as far as these could be recognized from the written material. We looked for symbol sense strategies, like recognition of key features, decomposition in sub-formulas, and reasoning and for other strategies, like 
making calculations (derivatives, and/or points). In the type B tasks, we also registered whether making a graph or a relevant part of the graph was used, as these were considered symbol sense strategies. When, in these type B tasks, symbol sense strategies were used, a strategy score of 1 was given. However, when calculations were made, the strategy score was 0 . The sum of these scores resulted in a StratTB score for each student. Besides the StratTB score, an effective strategy score (EffStratTB score) was also calculated, because using a symbol sense strategy did not guarantee a correct solution. When the symbol sense strategy resulted in a score of 0.5 or higher on a task, the effective strategy score was 1 . The sum resulted in an EffStratTB score for each student.

The scores on the written test, TA, TB, StratTB, and EffStratTB scores, are considered to be related to the general mathematics ability of the students, and the general mathematics ability of each of the 114 students was rated by their own teacher on a scale from 1 to 10 (called Math rating). In a one-way independent Anova on the students' Math ratings, no significant differences between the six schools were found.

In addition to the strategy scores from the written tests, we wanted a more detailed picture of the relation between the symbol sense use in the graphing tasks and in the algebra tasks (sub-question 2). As we expected that symbol sense involved in graphing formulas might be used in solving algebra tasks, we asked six students who belonged to the group of $25 \%$ highest scoring students on the graphing tasks (scores of 3 to 7.5 out of max 8) to think aloud during the test. Two of these students had very high Math ratings ( $\mathrm{T}$ and $\mathrm{K}$ ), two had more than average Math ratings (A and $\mathrm{M}$ ), and two had average Math ratings (Y and I). As our aim is to teach symbol sense to all students, these six students were also involved in the teaching of graphing formulas by hand. Thinking aloud is not expected to disturb thinking processes and should give reliable information about problem-solving activities (Ericsson, 2006). The thinking-aloud protocols were transcribed.

\subsection{Data analysis}

The first sub-question was about the relation between the TA scores and the three scores on type B tasks (TB scores, StratTB scores, EffStratTB scores). The assumptions of regression, independent errors, homoscedasticity, normally distributed errors, and multicollinearity were met (Field, 2012). Because of the small number of items, the scores on the type B tasks were not normally distributed; therefore, bias corrected and accelerated bootstrap 95\% CIs are reported.

The Math ratings were related to the scores on the type B tasks. To explore the relation between TA scores and scores on type B tasks, we first used regression with the type B scores as dependent variables and the TA scores as independent variable. Then, the Math rating was added also as an independent variable, to explore the influence of the Math rating on the scores of the type B tasks.

To get a more detailed picture of the relation between the TA scores and the scores on the type B tasks, the group of 114 students were divided into four quartile groups, based on their TA score. The $25 \%$ students with the highest TA score formed the quartile group Q4, the second 25\% students the Q3 group, etc. The Q4 group included 16 of the 21 students involved in the teaching graphing formulas. All written type A tasks were analyzed on the main strategies, that is, the use of recognition/reasoning, making calculations, and "no answer at all" (blank). The written type B tasks were analyzed on the main strategies recognition/ reasoning, making a graph, making calculations, and blank. For each task and each group 
(Q4, Q3, Q2, Q1), the relative frequencies of the main strategies and also the mean scores of the four groups on the tasks were calculated.

The thinking-aloud protocols could detail the main strategies that were used to analyze the written tasks. To analyze the thinking-aloud protocols, these were transcribed, and the transcripts were cut into idea units, fragments that contained crucial steps of explanations (Schwarz \& Hershkowitz, 1999). These idea units were encoded using Drijvers et al.'s (2011) framework and descriptions of experts' strategies in graphing formulas (Kop et al., 2015). Drijvers et al.'s framework uses the following categories: taking global view, reasoning, and strategic work. To detail the symbol sense in the category global view, strategies involved in graphing formulas were used: recognition of function families, using knowledge of prototypical graphs and other characteristics of the function family, and recognition of key features, without (instantly) knowing other characteristics. The category strategic work was split up: considering one's strategy and monitoring and abandoning a representation (e.g., making a graph). Also, signs of lack of symbol sense were encoded; e.g., when time-consuming and error-prone algebraic calculations were used, while the problem could be solved with recognition and reasoning. This led to the following codebook for the type A and type B tasks that is explained in Table 1:

We give three examples to illustrate the encoding.

As a first example, we consider student $\mathrm{T}$ working on task 14 (type B); they considered their strategy (S1), recognized the zeroes from the structure of the formula (R2), made a graph (S2), and used qualitative reasoning when $y$ values are described in terms of positive/negative $(\mathrm{Q})$ :

$\mathrm{Hmm}$, not nice to expand the brackets and to differentiate the function; but can it be done smarter? (S1); we can say that there will be a zero at 0 , and when $14-2 x=0$, so, at 7 and at 4 (R2); what shape do we have? (S2); for large $x$ it is positive multiply negative multiply negative, so positive; for a very negative number we get a negative outcome (Q) (followed by a correct graph; score 1; encoding R2,Q,S1,S2).

As a second example, student A was working on task 4 (task B); they started with calculations (C), monitored their strategy (S1), recognized a key feature (asymptote) (R2), and used qualitative reasoning about function behavior in the neighborhood of $x=3(\mathrm{Q})$.

First expand the brackets $x^{2}-13 x+30+40 /(x-3)$ (C); can this be larger than 70 ?; I'm going to try to find the turning point (S1); then see whether it is a parabola with a max or something like that, but there is also a broken function; let us see whether it is a parabola (R1) and see whether turning point is beneath or above 70 and then $40 \ldots . . ?$ (S1) (tries to calculate (C)) No, this will not work (S1); I think I calculate some points (S1); there is a vertical asymptote at $x=3$ (R2); so, when $x-3$ is very small then this part becomes very large and dominate the rest of the function $(\mathrm{Q}) ; \ldots x-3$ can be infinity small and then the fraction will be very large and easily above $70(\mathrm{Q})$ (score 1; encoding: R2,Q,S1,C) 
Table 1 Encoding the thinking-aloud protocols

\begin{tabular}{lll}
\hline Code category & Code & Description \\
\hline Recognition & R1 & $\begin{array}{c}\text { Recognizing a function family (families) and using prototypical } \\
\text { graphs and/or other characteristics of the function family } \\
\text { Reasoning }\end{array}$ \\
Q Q 2 & $\begin{array}{c}\text { Recognizing and using key graph feature(s) (e.g. a vertical asymptote, } \\
\text { zeroes, etc.) } \\
\text { (Qualitative) reasoning about e.g. parts of graph (infinity behavior, } \\
\text { in/decreasing, positive/negative, etc.), that is, using global } \\
\text { descriptions (e.g., "a square root translated to the right"), ignoring } \\
\text { what is not relevant in the situation }\end{array}$ \\
Calculation (as an indication of \\
lack of symbol sense)
\end{tabular}

In example 3, student $\mathrm{Y}$ was working on task 3 (task B); they used graphs (S2) and prototypical graphs (R1) and described a "reversed" prototypical graph (Q).

$2^{x}$ is equation of $\mathrm{e}^{x}(\mathrm{R} 1)$, so goes above (sketches a graph (S2); $2^{-x}$ goes the other direction (Q), so, they have 1 point of intersection (two correct graphs; score 1; encoding R1,Q,S2)

The categories to describe symbol sense in the codebook show some similarities with the Pierce and Stacey (2004) algebraic expectation framework. However, because our focus is on reading through formulas and making sense of them, the manipulation of formulas and equivalence of formulas plays a minor role compared with the Pierce and Stacey framework. The encoding was used to qualitatively study similarities and differences between the symbol sense use in the graphing and algebra tasks of each student.

\section{Results}

First, Table 2 shows the correlation between the variables Math rating, TA scores, TB, StratTB, and EffStratTB scores. The TA scores were strong correlated with the type B scores.

Next, the Math rating was added as an independent variable in the regression model with TB score as dependent variable and TA score as independent variable and later also with the StratTB score and the EffStratTB score as dependent variables. Table 3 shows that this resulted in slightly higher correlation coefficients, .694, .543, and .639, respectively, than were found in Table 2.

Table 2 Pearson's correlation coefficients between variables, with 95\% bias corrected and accelerated confidence intervals

\begin{tabular}{lllll}
\hline & TA score & TB score & StratTB score & EffStratTB score \\
\hline Math rating & $.324[.147, .493]$ & $.479[.318, .623]$ & $.305[.115, .470]$ & $.424[.245, .574]$ \\
TA score & & $.630[.492, .756]$ & $.514[.372, .646]$ & $.590[.438, .719]$ \\
TB score & & $.689[.598, .767]$ & $.921[.888, .945]$ \\
StratTB score & & & $.708[.619, .781]$ \\
\hline
\end{tabular}

All correlations are significant $(p<.001)$ 
Table 3 Linear model of predictors of type B scores, with $95 \%$ bias corrected and accelerated confidence intervals

\begin{tabular}{lllllll}
\hline Dependent variable & & SE B & Partial correlations & $\beta$ & $p$ \\
\hline TB scores & Constant & $-1.40[-3.00, .08]$ & .72 & & & .054 \\
& TA score & $.68[.48, .90]$ & .09 & .57 & .53 & $.000^{* * * *}$ \\
& Math rating & $.49[.24, .75]$ & .11 & .38 & .31 & $.000^{* * * *}$ \\
StartTB scores & Constant & $1.52[-.53,4.08]$ & 1.12 & & .180 \\
& TA score & $.79[.49,1.14]$ & .15 & .46 & .46 & $.000^{* * * *}$ \\
& Math rating & $.32[-.10, .66]$ & .18 & .17 & .15 & .071 \\
EffStratTB scores & Constant & $-1.40[-2.65, .07]$ & .68 & & $.041^{*}$ \\
& TA score & $.57[.37, .80]$ & .09 & .53 & .51 & $.000^{* * * *}$ \\
& Math rating & $.36[.13, .57]$ & .11 & .31 & .26 & $.001^{* * *}$ \\
\hline
\end{tabular}

${ }^{*} p<.05,{ }^{* *} p<.01,{ }^{* * *} p<.001$

\subsection{Students' symbol sense in type A and type B tasks}

Tables 4 and 5 show a more detailed picture of strategies of the Q4 and Q3 groups on the selection of type A and B tasks. The students in the groups Q1 and Q2 scored much lower on the use of symbol sense strategies than the Q3 and Q4 groups. Therefore, we only report on the Q3 and Q4 groups for a representative set of tasks. For type A tasks, we choose task 9 and 11 (working with parameters), task 15 (finding a formula), task 16 (graphing a formula), and task 19 (checking features of a graph). For type B tasks, we choose task 2 and 3 (number of solutions), task $4(y>70)$, task 5 (inequality), task 7 ( $y$ values), task 14 (about maximum), and task 18 (reasoning from formula). Tables 4 and 5 show that students of group Q4, as expected, used more symbol sense strategies than those in group Q3. See Tables 4 and 5.

In the other tasks that are not included in Table 5, also, the Q4 students used more symbol sense than the Q3 students, who at their turn did much better than the groups Q1 and Q2. The Q4 students also had higher mean scores than the Q3 group. Sometimes the differences in mean scores were very large, e.g., on task 1 (.57 vs $.18)$, task 2 (.47 vs .13), task 3 (.49 vs .17), task 4 (.33 vs .16), task 7 (.84 vs .50), task 13 (.45 vs .22), and task 14 (.30 vs .00). However, we found exceptions, namely, the tasks about inequalities (task 5 and 6). In these tasks, the Q3 students used symbol sense strategies more often than the Q4 students, and in task 5, the inequality $x(x-1)>4 x$, the Q3 students scored higher than the Q4 students (mean scores 0.70 versus 0.57 ). In the discussion, we discuss these findings about the inequalities.

Table 4 Strategy use (in percentages) of group Q3 and group Q4 on a selection of type A tasks

\begin{tabular}{|c|c|c|c|c|c|c|c|c|c|c|}
\hline \multirow[t]{2}{*}{ Strategy } & \multicolumn{2}{|c|}{ Task 9} & \multicolumn{2}{|c|}{ Task 11} & \multicolumn{2}{|c|}{ Task 15} & \multicolumn{2}{|c|}{ Task 16} & \multicolumn{2}{|c|}{ Task 19} \\
\hline & Q3 & Q4 & Q3 & Q4 & Q3 & Q4 & Q3 & Q4 & Q3 & Q4 \\
\hline Blank & 36 & 12 & 25 & 8 & 71 & 35 & 68 & 35 & 57 & 15 \\
\hline Calculation & 14 & 19 & 18 & 4 & 7 & 8 & 11 & 4 & 0 & 0 \\
\hline Recognition reasoning & 50 & 69 & 57 & 89 & 18 & 58 & 21 & 61 & 43 & 85 \\
\hline
\end{tabular}


Table 5 Strategy use (in percentages) of group Q3 and group Q4 on a selection of type B tasks

\begin{tabular}{|c|c|c|c|c|c|c|c|c|c|c|c|c|c|c|}
\hline \multirow[t]{2}{*}{ Strategy } & \multicolumn{2}{|c|}{ Task 2} & \multicolumn{2}{|c|}{ Task 3} & \multicolumn{2}{|c|}{ Task 4} & \multicolumn{2}{|c|}{ Task 5} & \multicolumn{2}{|c|}{ Task 8} & \multicolumn{2}{|c|}{ Task 14} & \multicolumn{2}{|c|}{ Task 18} \\
\hline & Q3 & Q4 & Q3 & Q4 & Q3 & Q4 & Q3 & Q4 & Q3 & Q4 & Q3 & Q4 & Q3 & Q4 \\
\hline Blank & 25 & 12 & 39 & 31 & 14 & 12 & 7 & 0 & 14 & 8 & 46 & 23 & 79 & 31 \\
\hline Calculation & 36 & 27 & 18 & 19 & 36 & 15 & 14 & 50 & 4 & 0 & 39 & 27 & 4 & 4 \\
\hline Making graph & 25 & 46 & 25 & 46 & 0 & 0 & 11 & 12 & 0 & 0 & 4 & 27 & 0 & 0 \\
\hline Recognition reasoning & 14 & 15 & 18 & 4 & 50 & 73 & 68 & 39 & 82 & 92 & 11 & 23 & 18 & 65 \\
\hline
\end{tabular}

To qualitatively back up the quantitative findings, the thinking-aloud protocols were analyzed according to the codebook of Table 1 . Results of these analyses are presented in Table 6. In the columns "symbol sense on graphing tasks" and "symbol sense in algebra tasks," the strategies that were predominantly used by a student are reported, that is, strategies used in more than $30 \%$ of the tasks. In Table 6, we used the following codes: $\mathrm{R} 1$, recognition of function families; R2, recognition of key graph features; Q, qualitative reasoning; S1, considering a strategy and monitoring; S2, abandoning a representation (e.g., making a graph); and $\mathrm{C}$, calculation.

The results in Table 6 seem to confirm the findings of the quantitative analyses of Tables 2 and 3, showing a relation between the scores on the graphing and algebra tasks. Table 6 shows that students often used recognition and qualitative reasoning when working on both kinds of tasks. As expected, the S2 strategy (abandoning a representation) was used more often in the algebra tasks than in the graphing tasks. Apart from the S2 strategy, there was some relation between the strategies used in both types of tasks; only students $\mathrm{A}$ and $\mathrm{M}$ showed a larger difference in strategy use between both kinds of tasks, as they often started with calculations working on the algebra tasks. In Appendix 2, for each student, illustrative transcripts with encodings plus samples of their written work are given. It shows that, in both tasks, the students often needed combinations of recognition, reasoning, and strategic work to solve the tasks. However, using more strategies was not always an indication of proficiency. For instance, the high achieving student $\mathrm{K}$ was short in their reasoning, using function families and qualitative reasoning.

Table 6 Scores and strategy use of the six thinking-aloud students

\begin{tabular}{lllll}
\hline Student & $\begin{array}{l}\text { Total score on } \\
\text { graphing tasks }(\max 8)\end{array}$ & $\begin{array}{l}\text { Symbol sense on } \\
\text { graphing tasks (type A) }\end{array}$ & $\begin{array}{l}\text { Total score on algebra } \\
\text { tasks (max 12) }\end{array}$ & $\begin{array}{l}\text { Symbol sense on } \\
\text { algebra tasks (type B) }\end{array}$ \\
\hline $\mathrm{T}$ & 7.5 & $\mathrm{R} 1, \mathrm{R} 2, \mathrm{Q}$ & 7.7 & $\mathrm{R} 1, \mathrm{R} 2, \mathrm{Q}, \mathrm{S} 1, \mathrm{~S} 2$ \\
$\mathrm{~A}$ & 6.2 & $\mathrm{R} 1, \mathrm{R} 2, \mathrm{Q}$ & 7.0 & $\mathrm{R} 1, \mathrm{Q}, \mathrm{C}$ \\
$\mathrm{Y}$ & 4.7 & $\mathrm{R} 1, \mathrm{R} 2, \mathrm{Q}$ & $\mathrm{R} 1, \mathrm{Q}, \mathrm{S} 2$ \\
$\mathrm{I}$ & 3.0 & $\mathrm{R} 2, \mathrm{Q}, \mathrm{C}$ & 4.5 & $\mathrm{Q}, \mathrm{C}$ \\
$\mathrm{K}$ & 5.0 & $\mathrm{R} 1, \mathrm{Q}$ & 4.5 & $\mathrm{R} 1, \mathrm{Q}, \mathrm{S} 2$ \\
$\mathrm{M}$ & 3.7 & $\mathrm{R} 1, \mathrm{R} 2, \mathrm{Q}$ & 8.0 & $\mathrm{Q}, \mathrm{C}$ \\
\hline
\end{tabular}




\section{Discussion and conclusion}

In this study, we investigated how students' graphing by hand abilities might be related to symbol sense abilities to solve non-routine algebra tasks. We designed a symbol sense test with graphing problems (type A tasks) and other algebra tasks that could be solved with graphs and reasoning, without algebraic calculation (type B tasks).

With respect to the first sub-question about the relation between the graphing formulas abilities and the abilities to solve the algebra problems with symbol sense, we found that the students who scored better on the graphing tasks also scored higher on the algebra tasks. General mathematics abilities might explain this relation. However, when Math rating was added as an independent variable, the explained variance of the scores on the algebra tasks hardly increased. This suggested a positive relationship between students' graphing abilities and their abilities to solve algebra tasks. A similar positive relationship was found between the scores on the graphing tasks and the symbol sense scores on the algebra tasks (StratTB and EffStratTB scores), indicating that students who scored higher on the graphing tasks used more and more effectively symbol sense strategies while solving the algebra tasks. This relation was confirmed by the analyses between the Q3 and Q4 groups in Tables 4 and 5, which showed that the Q4 students used more symbol sense strategies than the Q3 students.

The second sub-question was about similarities and differences between symbol sense use in the graphing and algebra tasks. In the analyses of the thinking-aloud protocols, we found that the six students often used similar symbol sense strategies in both the graphing and algebra tasks. Students' approaches to solve the graphing tasks could be described through combinations of recognition of function families and using prototypical graphs and characteristics, recognition of key features of the function, and qualitative reasoning. To these combinations, the strategy "abandoning a representation" (making a graph) was added, when working on the algebra tasks. The high-scoring students more often used "making a graph" and had a larger repertoire of symbol sense strategies than the other students, who more often tried to use calculations, and had trouble to use combinations of strategies. The findings suggest that, besides "make a graph," students often used similar strategies in the graphing and algebra tasks.

The study aimed to contribute to the knowledge of symbol sense and to the students' symbol sense abilities in graphing formulas and in non-routine algebra tasks. With respect to the main research question on how students' graphing by hand abilities might be related to their symbol sense use while solving non-routine algebra tasks, our findings suggest that students could use their symbol sense involved in graphing formulas, that is, a combination of recognition of function families and graph features from the structure of the function, qualitative reasoning, and strategic work, to solve algebra tasks.

\subsection{Limitations}

Before discussing these results in more detail, we acknowledge that the study, of course, also came with limitations. The algebra tasks in our test were restricted to problems, predominantly using the variables $x$ and $y$, that could be solved with graphs and reasoning, without algebraic calculation. Another issue is the combination of graphing and algebra tasks in one test which might have given suggestions to use graphs in the type B tasks. In a future study, these issues could be addressed by omitting explicit graphing tasks, by also using other variables than $x$ and $y$, and by adding some tasks that need some algebraic calculation. In this article, the focus was 
on the relation between graphing abilities and the symbol sense abilities to solve algebra tasks. A next step would be to set up a quasi-experimental study, in which a group of students were taught to graph formulas by hand, using a control group and a pre-test and post-test.

In discussing the findings, we note that the results of this study seem to confirm earlier research about the problems Dutch students have with algebra: students have problems graphing formulas by hand (Kop et al., 2020) and with identifying and using the structure of algebraic expressions (Van Stiphout, Drijvers, \& Gravemeijer, 2013). Regular teaching of algebra does not seem to develop these aspects of symbol sense. Although only Dutch students were involved in this study, literature about symbol sense (Arcavi et al., 2017; Drijvers et al., 2011; Kieran, 2006; Arcavi, 1994; Ayalon, Watson, \& Lerman, 2015; Hoch \& Dreyfus, 2005, 2010; Oehrtman et al., 2008) and personal conversations with teachers and scholars from other countries suggest that grade 12 students abroad have similar problems with symbol sense.

A remarkable finding, described in the "Results" section, was that in task 5, the inequality, the Q3 students scored higher and did use more symbol sense strategies than the Q4 students, who more often tried calculations to solve this task. We wonder why the Q4 students did not use their graphing skills in this task, as we expect that they could easily graph both formulas. Although we know from literature that students may over rely on the symbolic representation even when graphs are more appropriate (Knuth, 2000; Eisenberg \& Dreyfus, 1994; Kenney, 2008; Slavit, 1997), we assume that the inequality triggered previously learned associations that hinder later learned symbol sense, as was suggested by student Y (see Appendix 2).

\subsection{Implications}

The findings of the current study suggest a positive relationship between the ability to graph formulas by hand and to solve non-routine algebra tasks and showed similarities in the symbol sense used in both kinds of tasks. The contribution of this study is that it describes this symbol sense through combinations of recognition of function families and key graph features from the structure of the formulas, qualitative reasoning, and strategic work and that it suggests how this symbol sense might be taught to students.

Graphing formulas and covariational reasoning, in the context of formulas, are related as both have a focus on global (qualitative) graphs. In this study, it is explicitly shown how and what qualitative reasoning was used by students. The importance of qualitative reasoning and its omission in regular mathematics education have been stressed by Goldenberg, Lewis, and O'Keefe (1992), Yerushalmy (1997), and Duval (2006). In their elaborations about covariational reasoning, Moore and Thompson (2015) have problematized what they called static shape thinking that is seeing a graph-as-a-wire. However, our findings show that the students successfully used prototypical graphs of function families as building blocks in their reasoning, when working on the graphing tasks and using the strategy "making a graph" in the algebra tasks. The need for such repertoire of functions that can be instantly visualized by a graph has been stressed by many, for example, by Eisenberg and Dreyfus (1994), Stylianou and Silver (2004), and Duval (2006). In combination with qualitative reasoning, such repertoire might provide a knowledge base that is needed to enable students using visualizations to solve algebra problems ("making a graph" strategy). Visualization is more than just making or perceiving graphs, it is about noticing and understanding the whole that is represented with its features, for which a solid knowledge base is needed (Duval, 2006).

Teaching symbol sense is not easy (Arcavi et al., 2017; Hoch \& Dreyfus, 2005). Before we describe our suggestions about teaching symbol sense, we discuss extant approaches. Pierce 
and Stacey (2007) suggested highlighting the formula's structure and key features in classroom discussions when working with graphs. Friedlander and Arcavi (2012) focused on meaningful reading of algebraic formulas and formulated small tasks that focused on, e.g., qualitative thinking and global comprehension. Kindt (2011) gave many examples of productive practice in algebra. These activities are valuable and can be easily added to existing lessons, but often manipulations of formulas play a central role in these activities, and they lack a systematic and a step-by-step development. Our approach to teaching symbol sense focuses on enabling students to make sense of formulas and to read through formulas. In literature, it has been suggested that giving meaning to formulas can be done via linking representations of functions and/or via realistic contexts (Kieran, 2006). However, except for linear and exponential functions, formulas often cannot be directly linked to realistic contexts. Therefore, we choose to link formulas to graphs through graphing formulas. To learn about functions, many have recommended to use technology to link representations (Kieran, 2006; Kieran \& Drijvers, 2006; Heid, Thomas, \& Zbiek, 2013). However, Goldenberg (1988) found that students established the connection between formula and graph more effectively when they drew graphs by hand than when they only performed computer graphing. Others have recognized the need for pen-and-paper activities when working with technology (Arcavi et al., 2017; Kieran \& Drijvers, 2006). Therefore, we tried to promote students' symbol sense through graphing formulas by hand.

In this study, we found strong correlations between students' graphing by hand abilities and their abilities to solve algebra tasks and their use of symbol sense while solving non-routine algebra tasks. These correlations could not be accounted for by students' general mathematics abilities. Symbol sense involved in graphing formulas includes combinations of recognition of function families and key features from the structure of formulas and (qualitative) reasoning, and it is a subset of symbol sense involved in solving non-routine algebra tasks. In the current study, 16 of the 21 students who were involved in the teaching of graphing formulas by hand belonged to the 25\% highest scoring students on the graphing tasks (Q4 group), who used more symbol sense when solving algebra tasks than the other students. The six thinkingaloud students, all involved in the teaching and belonging to the Q4 group, showed that they were able to use their symbol sense and graphing formulas abilities to solve the non-routine algebra tasks, that is, combinations of recognition, qualitative reasoning, and strategic work. This suggests that teaching symbol sense in the domain of graphing formulas by hand might be an effective means to teach essential aspects of symbol sense involved in solving non-routine algebra tasks. In a previous study, we showed how to teach graphing formulas by hand, using these essential aspects of symbol sense (Kop et al., 2020).

The current study provides more insight in the relation between symbol sense involved in graphing formulas by hand and in solving non-routine algebra tasks, in what aspects of symbol sense students can use while solving non-routine algebra tasks, and how this symbol sense might be taught. However, more research is needed to investigate how educational practices might benefit from these insights. Also, lower grades of secondary school could be included in such research, investigating Ruthven's suggestion to start algebra with graphing activities, instead of algebraic calculations (Ruthven, 1990), and our suggestion to learn about functions through a combination of graphing tools to explore functions and graphing by hand activities to foster students' symbol sense. 


\section{Appendix 1: Symbol sense test}

Tasks $9,10,11,15,16,17,19$, and 20 are the graphing tasks (type A tasks).

1) Give the number of zeroes of the function $f(x)=x\left(x^{2}+2\right)-12$
A. no zeroes
B. one zero
C. two zeroes
D. three zeroes
E. more than three zeroes

2) Give the number of solutions of the equation: $5 \ln (x)=\frac{1}{2} x-10$

3) Give the number of solutions of the equation: $2^{x}=2^{-x}+3$

4) Can the $y$-value of $y=-0.1(x-3)(x-10)+40 /(x-3)$ become larger than 70 ?

5) Solve the inequality: $x(x-1)>4 x$

6) Solve the inequality: $\frac{x^{2}-4}{x^{2}-9}<0$

7) What outcome(s) can $y$ have when $y=24-0,01(x+5)^{4}$ ?

8) When $x$ is very large, the function $f(x)=\left(3 \mathrm{e}^{-x^{2}}+x^{2}\right)^{3}+\frac{70}{x^{4}}$ can be approximated by:

Choose the best alternative out of: A. $y=x^{6}$; B. $y=x^{5}$; C. $y=70 x^{-4} ;$ D. $y=27 \mathrm{e}^{-3 x^{2}}$;

E. none of these

9) Here is a graph of $f_{p}(x)=x^{4}-p x^{2}$ for $p=1$.

Make a sketch of the graph of $f_{p}$ for a value $p>1$.

Explain your answer.

10) Here is a graph of $f_{p}(x)=\mathrm{e}^{-x^{2}+p}$ for $p=1$.

Make a sketch of the graph of $f_{p}$ for a

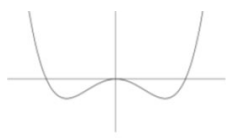
value $p>1$.

Explain your answer.

11) Here is a graph of $f_{p}(x)=(x-p)^{2}+2 p$ for $p=1$.

Make a sketch of the graph of $f_{p}$ for a value $p$ $>1$.

Explain your answer.

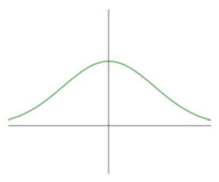


12) Consider for each value of $p$ the equation $2 x^{3}=p x+1$.

How many solutions can this equation have?

13) The number of different species of animals $\mathrm{A}$ in a domain can be modeled with the function $A=\frac{300}{2+3 \cdot 0.87^{t}} ; t=0$ is the year 2000 . What does this formula tell about the number of different species in the domain?

14) Choose the correct alternative: A maximum of the function $y=x(14-2 x)(8-2 x)$ is situated in A. [-4;0]; B: [0;4]; C: [4;7]; D: [7;14]

15) Find a formula that fits the graph.

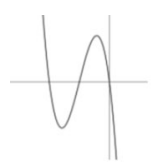

16) Make a sketch of the graph of $y=4 x \sqrt{x+5}$

17) Make a sketch of the graph of $y=3^{x}+5 x^{-2}$

18) The formula $C=0,13\left(1,92^{-t}-1,92^{-6 t}\right)$ gives information about the concentration of medicine in $\mathrm{mg} / \mathrm{cm}^{3} ; t$ is the time in hours. What does this formula tell about the concentration $C$ ?

19) This is a part of the graph of $f(x)=\left(x^{2}-1\right)\left(x-\frac{3}{2}\right)$.

Do you miss some characteristic features of this function?

If yes, graph the whole graph.

20) This is a part of the graph of $f(x)=\frac{20 x^{4}}{x^{4}+2000}$ Do you miss some characteristic features of this function?

If yes, graph the whole graph.

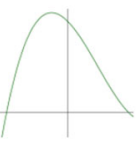




\section{Appendix 2: Transcript of thinking-aloud protocols with encodings plus samples from student work}

To portray the students' symbol sense (or lack of symbol sense), we selected for each student representative fragments of their thinking-aloud protocols about a certain task, combined with samples of their written work of that task.

Student $\mathrm{T}$ is a high-achieving student who used a broad repertoire of symbol sense strategies, including scanning and monitoring (S1); see example of task 14 in "Data analysis" section), and hardly used calculations.

Student $\mathrm{T}$ working on task 1 :

Hmmm, $x^{2}+2$ is just a normal parabola, with a minimum, 2 above (R1); multiplied by $x$; if we would take irrational numbers (complex numbers?), then we have 3 ; but when you multiply it (the parabola) with $x$, then right positive and left negative; then you would have only 1

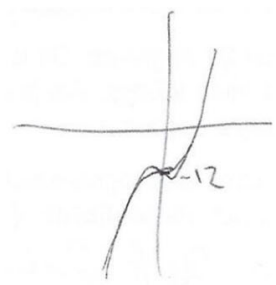
zero (Q); it goes like this (gestured a prototypical $x^{3}$ graph (S2)), and that - 12; I think there is only 1 zero (correct graph; score 1; encoding: R1,Q,S2)

Student $\mathrm{T}$ working on task 3 :

$2^{x}$ goes like this (S2, R1); $2^{-x}$ goes like this, and 3 higher (R1); so, 1 solution (score 1; encoding R1, S2)

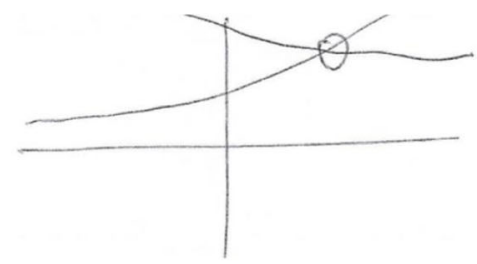

Student T working on task 9:

So, $x-1.5$; we have a zero, a zero at $x=1.5(\mathrm{R} 2)$; and zeroes at $x^{2}$ give zero at $x=1$; and ..; probably, this zero is at 1 and $-1(\mathrm{R} 2)$; then it goes further and is there a zero at 1.5 , I guess; let's check what is happening at large $x$-values? $y$ becomes positive (Q); so, (zero at) $x=1.5$ has to be added (sketches a correct graph; score 1; encoding R2,Q) Student $\mathrm{T}$ working on task 12 :

$x^{3}$ has this shape (sketches graph) (S2,R1); equals $p x+1$, which goes like this, or like this, or ...(R1,Q); it can have 3 solutions, because it goes through $(0,1)$; I think it can have up to 3 solutions (score 0.7; encoding R1,Q,S2) 
Student T working on task 15:

A degree 3 and 2, and at $x=0$, it has to

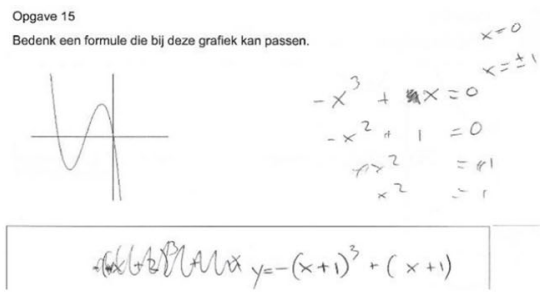
be 0 ; we have a $x^{3}(\mathrm{R} 1)$, but it has to be translated to the left $(\mathrm{Q})$; this is the midpoint of $x^{3}$, and then it has to be negative: $-x^{3}(\mathrm{R} 2)$; then add a linear function, so that at $x=0$ it is 0 (checks at $x=2$ and considers a translation +8 , but then focuses on the zeroes and calculates the zeroes, and translates the graph 1 to the left) (C) (score 1; encoding R1,R2,Q,C)

Student T working on task 18:

When $t$ is increasing then this $\left(1,92^{-t}\right)$ is becoming small (R2), and the other $\left(1,92^{-6 t}\right)$ even becomes faster small (R2, Q), because it is a negative number (in the power),..., does not matter, it just becomes very small and is decreasing (Q) (they does not pay attention to increasing part at the start; score 0.5; encoding: R2,Q)

Student A often started with calculation in the algebra tasks, but monitored their progress, and then used recognition and reasoning (see also example of task 4 in data analysis section) Student A working on task 2:

How can I find zeroes? (S1)I try to solve it. I think because it is not quadratic $\ldots ; 5 \ln (x)-\frac{1}{2} x+10(\mathrm{~S} 2) \ldots$; first calculate the derivative: $\frac{5}{x}-\frac{1}{2}(\mathrm{C})$ and searching for turning point; equals 0 , so, $x=10$; there is a turning point at $x=10$ and when we substitute 10 then we get left $5 \ldots$; we get two zeroes (writes $x=10 \rightarrow 1$ turning point, so two zeroes; score 0.7 ; encoding: S1,S2,C)

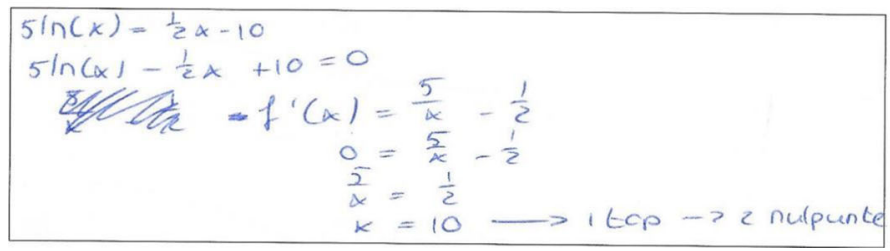


Student A working on task 5:

I think I first divide by $x(\mathrm{C})$ because then it becomes much easier; so, $x-1>4$; then it is very easy; so, $x>5$
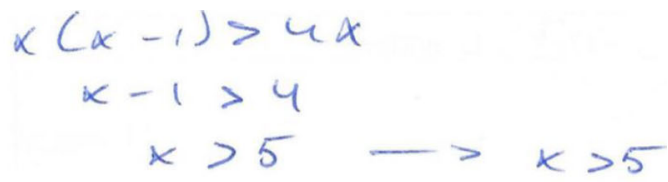

(score 0.3 ; encoding C)

Student A working on task 15:

I see two turning points and three zeroes; zero at $x=0$, and let's say, at $x=-2$ and $x=-4$; so, something like $x(x+2)(x+4)(\mathrm{R} 2)$; yes, then the turning points should be there; then taking care that when $x$ is positive the formula-outcomes become negative; then we need $-x(\mathrm{R} 2)$; checks at $\mathrm{x}=-5$ that $y$-value is positive) ( $\mathrm{S} 1)$ (correct formula $y=-x(x+2)(x+4)$; score 1; encoding R2,Q,S1)

Student $\mathrm{Y}$ is a hard-working student who thinks mathematics is difficult. They used often the strategy "make a graph" when solving algebra tasks (see example of task 3 in "Data analysis" section. Their work on inequalities suggested that previous learned procedures can give gave trouble (see task 5).

Student Y working on task 5:

I have to think about inequality-sign; when dividing or multiplying by - or + it turns; but I don't remember this (S1); I think when dividing; but I'm not sure; I divide by $x$, so, $x-1<4$, that means it is true for $x=5$; to check: substitute 5 gives $20(\mathrm{~S} 1)$; $>$ then larger or equal is not correct; when substituting, I get 20, but I do not know how to proceed (S1); I think the inequality sign reverses, but I'm not sure. (writes 20>20, not possible?; score 0; encoding: S1)
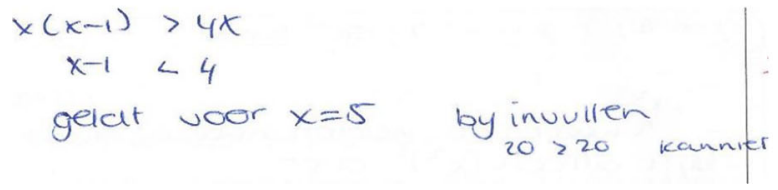

Student $\mathrm{Y}$ working on task 8:

When $x$ is very large then $70 / x^{4}$ fades because it becomes very small, approaches 0 (Q); therefore alternative $\mathrm{C}$ is not correct; when $x$ is very large then it becomes $y=$ $x^{6} ; \mathrm{e}^{- \text {large }}$ becomes almost 0 , so, when substituting something very large in $\mathrm{e}^{-x^{2}}$ it approaches to $0(\mathrm{Q})$; then only $\left(x^{2}\right)^{3}$ is left; I doubt 5 or 6 , but it is multiplying, so answer A (score 1; encoding: Q) 
Student Y working on task 11:

$(x-1)$ therefore a translation 1 to the right (R2); turning point is $(1,2)$; when $p$ is changing then it translates further; so, when we take $p=2$, then turning point is about here (R2) (sketches a correct parabola and give the coordinates of the turning

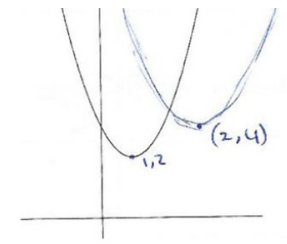
point: $(2,4)$; score 1; encoding: R2)

Student I thinks mathematics is very hard, but after the lessons about graphing formulas, student I developed more confidence in their mathematical thinking.

Student I working on task 2:

So, this is a long time ago. How do I do $5 \ln (x)=\frac{1}{2} x-10$
$e \log \left(x^{5}\right)=\frac{1}{2} x-10$
$e^{\frac{1}{2} x-10}=x^{5} \neq-$ this?(S1) I transform this equation: $\log _{\mathrm{e}}\left(x^{5}\right)=$ $\frac{1}{2} x-10(\mathrm{C}) \ldots$ Can I solve this equation? (S1) I can transform it into $x^{5}=\mathrm{e}^{\frac{1}{2} x-10}(\mathrm{C})$ but do I make any progress? ... $x^{5}$ can only be positive or negative (R1). No, I do not know (score 0; encoding: R1,C,S1)

Student I working on task 7:

$y$ cannot be larger than 24 because it is 'to the power 4' function (R1); then it is always positive, that is $0.01(x+5)^{4}$ is always positive $(\mathrm{Q})$, so $y$ cannot be larger than 24 ; so, $y \leq 24$ (score 1; encoding R1,Q)

Student I working on task 14:

What happens when I make $x$ very large: $14-2 x$ negative, $8-2 x$ negative, so, positive times negative is negative $(\mathrm{Q})$; that you don't want; when $x$ very negative, $14-2 x$ very positive, times very positive, then ....;no; I expand brackets (of $(14-2 x)(8-2 x))(C)$, dividing by $\mathrm{x}$; dividing by 4 ; (solves the equation $x^{2}-11 x+28=0$, finds $x=4$ and $\left.x=7\right)(C)$; so, turning point between 4 and 7 (score 0 ; encoding $\mathrm{Q}, \mathrm{C}$ ) 


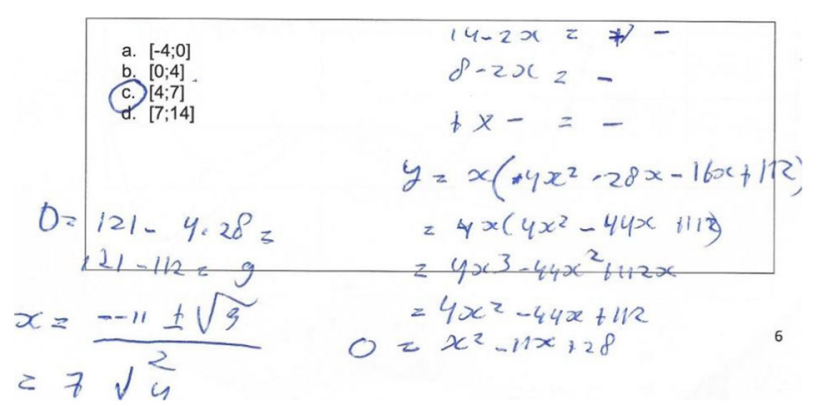

Student I working on task 16:

$4 x$ goes like this (R1); $\sqrt{x}$ like this (R1); $x+5$ means that it starts at $x=-5$ (sketches $\sqrt{x+5})(\mathrm{R} 1)$; here it is 0 (points at $\mathrm{x}=0$ ); here negative; I multiply these graphs; here it is positive (Q); I'm not sure

(score 1; encoding R1, Q)

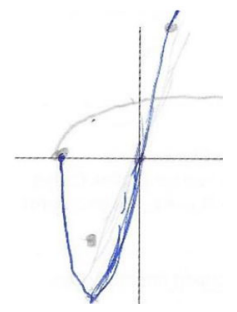

Student I working on task 19:

$\left(x^{2}-1\right)(x-1.5)$ gives $x^{3}-1.5 x^{2}-x+1.5(C) ; \ldots$ turning point in view; no, when $\mathrm{x}$ is larger then $x^{3}$ larger but $-x^{2}$ larger, finally it will be negative $(\mathrm{Q})$; so, all features in view: zeroes and y-values become negative when $x$ is larger (score 0 ) (score 0; encoding: C)

Student $\mathrm{K}$ is a high-achieving student who often used their repertoire of function families and qualitative reasoning and hardly used any calculations.

Student K working on task 2:

$\ln (x)$, so, e in the power something (gestures a correct graph) (R1,S2); $\frac{1}{2} x-10$ runs like this (gesture) (R1); so, 2 solutions (two correct graphs, score 1; encoding R1,S2) Student K working on task 10:

When $p$ larger then....it is $\mathrm{e}^{p} \cdot \mathrm{e}^{-x^{2}}(\mathrm{~S} 2)$, so it is multiplied by larger factor (Q), multiplying relative to $x$-axis (R1) (sketches a correct graph; score 1; encoding $\mathrm{R} 1, \mathrm{Q}, \mathrm{S} 2)$

Student M did not use their abilities to graph formulas to solve the algebra tasks; instead, they often started with calculations.

Student M working on 3:

A " $x$ " in the power; the +3 makes $2^{x}$ has to be larger than the $2^{-x} ; \ldots$; it makes a difference whether $x$ is positive or negative; look to the rules, with logarithm one gets 
$\ldots$; if you use both $2^{x}$ and $2^{-x} ; \ldots$; dividing them; you get $2^{2 x}=3(\mathrm{C})$, so one solution (not correct; score 0 ; encoding: $\mathrm{C}$ )

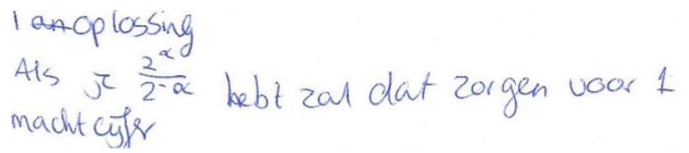

Student M working on task 8:

this $\left(70 / x^{4}\right)$ becomes very small so it faints $(\mathrm{Q})$; so, only consider the first part; $x^{2}$ very large; $-x^{2}$ very negative, so, $1 / \mathrm{e}^{x^{2}}$ becomes very small because $\mathrm{e}^{1000}$ very large (Q) so, it will be $x^{2}$ in the power 3; it will be in the power 6 (score 1; encoding: Q).

Student M working on task 17:

(writes $5 / x^{2}+3^{x}$ ) (S2); division, so $x$ cannot be 0 (R2); ... $3^{x}$ ever increasing (R1); the other $\left(5 / x^{2}\right)$ decreases to an asymptote (R2) and has only positive

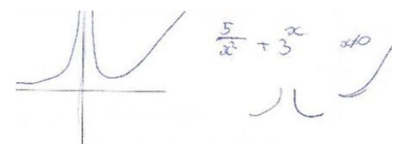
outcomes (Q)(sketches both sub-graphs); here, it is about $0+0(\mathrm{Q})$ and then it becomes very large towards the $y$-axis; on the other side of y-axis, the closer to $x=0$ the larger $y(\mathrm{Q})$ (sketches a correct graph; score 1; encoding R1,R2,Q,S2)

\section{Appendix 3: Description of intervention}

The intervention of five lessons of 90 min focused on learning to graph formulas, using recognition and reasoning. The teaching was based on design principles for learning complex skills (Kirschner \& Van Merriënboer, 2008), which were using a whole-task-first approach instead of a part-task approach, supporting students by providing help through modeling expert thinking, examples, sub-questions, and reflection questions, and the meta-heuristic "questioning the formula". This "Questioning the formula" reflects the levels of recognition used in expertise research in graphing formulas (Kop et al., 2015) and is about the habit of asking oneself questions like: "Do I instantly know the graph?", "Do I recognize a function family?", "Can I decompose the formula?", "Do I recognize graph features?", and "Can I do some strategic search for, for instance, infinity behavior?". Its importance has been stressed by, e.g., Pierce and Stacey (2007) and Landa (1983). Each day, there was a short plenary discussion ( $\max 10 \mathrm{~min}$ ) with some general feedback and reflection on the students' work and modeling of expert thinking processes. After the plenary, the students worked in pairs or groups of three, studied their personal feedback, and discussed strategies and solutions for the whole tasks. At the end of a lesson, all pairs and groups handed in their work for personal feedback. The tasks used in the teaching, formulated as whole tasks, reflected the "questioning 
the formula": task 1 and 2 concerned recognition of basic functions and aimed to develop a knowledge base of function families with their characteristic features and to deal with simple transformations; task 3 concerned the decomposition of formulas and the composition of subgraphs using qualitative reasoning; task 4 concerned the instant recognition of key graph features; and task 5 was about strategic exploration of parts of a graph, using qualitative reasoning. We now give more detailed information about the tasks.

Task 1 required students to match formulas of basic function $y=\sqrt{x}, y=x^{3}, y=0,5^{x}$, $y=\ln (x)$, and $y=|x|$ to their graphs. Task 2 was based on Swan (2005): Describe the differences and similarities between the graphs of the pairs of functions like $y=2 \sqrt{x}-4$ and $y=2 \sqrt{x-4}$ and $y=-3^{x}$ and $y=3^{-x}$. In task 3 , the function $y=\sqrt{x}(3 x-6)$ had to be graphed by multiplying the graphs of the sub-functions $y=\sqrt{x}$ and $y=3 x-6$. Task 4 was inspired by Burkhardt and Swan (2013) and Swan (2005) and concerned the recognition of graph features: What features of the given graph can be instantly read from the given two equivalent formulas $y=(x-4)^{2}-1$ and $y=(x-5)(x-3)$ ?

Task 5 concerned part-graph reasoning, using qualitative reasoning. For instance, what happens to the $y$ values of the functions $y=52.7 /\left(1+62,9 \cdot 0,692^{x}\right), y=0,6^{x} \cdot x^{60}$ when $x \rightarrow+\infty$ ? Choose $y \rightarrow+\infty ; y \rightarrow a \neq 0 ; y \rightarrow 0 ; y \rightarrow-\infty$

For each task, help was provided, and a reflection question was added. For instance, in task 2 (about recognizing transformations of basic functions), students could choose to use GeoGebra and/or to study worked-out examples for help. After each whole task, a reflection question was posed, in which students were asked to construct three new examples to demonstrate the principles of the whole task.

Open Access This article is licensed under a Creative Commons Attribution 4.0 International License, which permits use, sharing, adaptation, distribution and reproduction in any medium or format, as long as you give appropriate credit to the original author(s) and the source, provide a link to the Creative Commons licence, and indicate if changes were made. The images or other third party material in this article are included in the article's Creative Commons licence, unless indicated otherwise in a credit line to the material. If material is not included in the article's Creative Commons licence and your intended use is not permitted by statutory regulation or exceeds the permitted use, you will need to obtain permission directly from the copyright holder. To view a copy of this licence, visit http://creativecommons.org/licenses/by/4.0/.

\section{References}

Arcavi, A. (1994). Symbol sense: Informal sense-making in formal mathematics. For the Learning of Mathematics, 14(3), 24-35.

Arcavi, A. (2005). Developing and using symbol sense in mathematics. For the Learning of Mathematics, 25(2), $42-47$.

Arcavi, A., Drijvers, P., \& Stacey, K. (2017). The teaching and learning of algebra: Ideas, insights and activities. London / New York: Routledge.

Ayalon, M., Watson, A., \& Lerman, S. (2015). Functions represented as linear sequential data: Relationships between presentation and student responses. Educational Studies in Mathematics, 90(3), 321-339.

Bredeweg, B., \& Forbus, K. D. (2003). Qualitative modeling in education. AI Magazine, 24(4), 35-35.

Burkhardt, H., \& Swan, M. (2013). Task design for systemic improvement: Principles and frameworks. In C. Margolinas (Ed.), Task design in mathematics education: Proceedings of ICMI Study 22 (pp. 431-439). Oxford, UK: University of Oxford.

Carlson, M., Jacobs, S., Coe, E., Larsen, S., \& Hsu, E. (2002). Applying covariational reasoning while modeling dynamic events: A framework and a study. Journal for Research in Mathematics Education, 22(5), 352-378.

Carlson, M. P., Madison, B., \& West, R. D. (2015). A study of students' readiness to learn calculus. International Journal of Research in Undergraduate Mathematics Education, 1(2), 209-233. 
Drijvers, P., Goddijn, A., \& Kindt, M. (2011). Algebra education: Exploring topics and themes. In P. Drijvers (Ed.), Secondary algebra education. Revisiting topics and themes and exploring the unknown (pp. 5-26). Rotterdam, Boston, Taipei: Sense Publishers.

Duval, R. (1999). Representation, vision and visualization: Cognitive functions in mathematical thinking. Basic issues for learning. In F. Hitt \& M. Santos (Eds.), Proceedings of the 21st annual meeting of the North American Chapter of the International Group for the Psychology of Mathematics Education (pp. 3-26). Morelos, Mexico: Cuernavaca.

Duval, R. (2006). A cognitive analysis of problems of comprehension in a learning of mathematics. Educational Studies in Mathematics, 61(1-2), 103-131.

Eisenberg, T., \& Dreyfus, T. (1994). On understanding how students learn to visualize function transformations. In E. Dubinsky, A. Schoenfeld, \& J. Kaput (Eds.), Research in collegiate mathematics education (vol. 1, pp. 45-68). Providence, RI: American Mathematical Society.

Ericsson, K. A. (2006). Protocol analysis and expert thought: Concurrent verbalizations of thinking during experts' performance on representative tasks. In K. A. Ericsson, N. Charness, P. J. Feltovich, \& R. R. Hoffman (Eds.), The Cambridge handbook of expertise and expert performance (pp. 223-241). Cambridge, UK: Cambridge University Press.

Fey, J. T. (1990). Quantity. In L. A. Steen (Ed.), On the shoulders of giants: New approaches to numeracy (pp. 61-94). Washington, DC: National Academy Press.

Field, A. P. (2012). Discovering statistics using SPSS (4nd ed.). London, UK: Sage Publications Ltd.

Friedlander, A., \& Arcavi, A. (2012). How to practice it: An integrated approach to algebraic skills. Mathematics Teacher, 105(8), 608-614.

Goldenberg, E., Lewis, P., \& O'Keefe, J. (1992). Dynamic representation and the development of a process understanding of function. In G. Harel \& E. Dubinsky (Eds.), The concept of function: Aspects of epistemology and pedagogy (pp. 235-260). Washington, DC: Mathematical Association of America.

Goldenberg, E. P. (1988). Mathematics, metaphors, and human factors: Mathematical, technical, and pedagogical challenges in the educational use of graphical representation of functions. The Journal of Mathematical Behavior, 7(2), 135-173.

Heid, M. K., Thomas, M. O. J., \& Zbiek, R. M. (2013). How might computer algebra systems change the role of algebra in the school curriculum? In A. J. Bishop, M. A. Clements, C. Keitel, J. Kilpatrick, \& F. K. S. Leung (Eds.), Third international handbook of mathematics education (pp. 597-642). Dordrecht, the Netherlands: Springer.

Hoch, M., \& Dreyfus, T. (2005). Students' difficulties with applying a familiar formula in an unfamiliar context. In H. L. Chick \& J. L. Vincent (Eds.), Proceedings of the 29th Conference of the International Group for the Psychology of Mathematics Education (vol. 3, pp. 145-152). Melbourne, Australia: PME.

Hoch, M., \& Dreyfus, T. (2010). Developing Katy's algebraic structure sense. In V. Durand-Guerrier, S. SouryLavergne, \& F. Arzarello (Eds.), Proceedings of the 6th Congress of the European Society for Research in Mathematics (pp. 529-538). Lyon, France: CERME.

Janvier, C. (1987). Problems of representation in the teaching and learning mathematics. Hillsdale, NJ: Lawrence Erlbaum Associates.

Keller, B. J. (1994). Symbol sense and its development in two computer algebra system environments. (Western Michigan University, 1993). Dissertation Abstracts International, 54, 5704.

Kenney, R. (2008). The influence of symbols on pre-calculus students' problem solving goals and activities. $\mathrm{PhD}$ diss: North Carolina State University.

Kieran, C. (2006). Research on the learning and teaching of algebra. In A. Gutiérrez \& P. Boero (Eds.), Handbook of research on the psychology of mathematics education (pp. 11-49). Rotterdam, the Netherlands: Sense.

Kieran, C., \& Drijvers, P. (2006). The co-emergence of machine techniques, paper-and-pencil techniques, and theoretical reflection: A study of CAS use in secondary school algebra. International Journal of Computers for Mathematical Learning, 11(2), 205-263.

Kindt, M. (2011). Principles of practice. In P. Drijvers (Ed.), Secondary algebra education. Revisiting topics and themes and exploring the unknown (pp. 137-178). Rotterdam, Boston, Taipei: Sense Publishers.

Kirschner, P.A., \& Van Merriënboer, J.J.G. (2008). Ten steps to complex learning: A new approach to instruction and instructional design. In T. L. Good (Ed.), 21st Century Education: A Reference Handbook (pp. 244 253). Thousand Oaks, CA: Sage.

Knuth, E. J. (2000). Student understanding of the Cartesian connection: An exploratory study. Journal for Research in Mathematics Education, 31(4), 500-507.

Kop, P., Janssen, F., Drijvers, P., Veenman, M., \& Van Driel, J. (2015). Identifying a framework for graphing formulas from expert strategies. The Journal of Mathematical Behavior, 39, 121-134. 
Kop, P. M.G.M., Janssen, F. J.J.M., Drijvers, P. H.M., \& van Driel, J. H. (2020). Promoting insight into algebraic formulas through graphing by hand. Mathematical Thinking and Learning, 1-20. https://doi.org/10.1080 $/ 10986065.2020 .1765078$

Landa, L.N. (1983). Descriptive and prescriptive theories of learning and instruction: An analysis of their relationships and interactions. In C.M. Reigeluth (Ed.), Instructional-design theories and models: An overview of their current status (pp. 55-73). Hillsdale, NJ: Lawrence Erlbaum Associates.

Leinhardt, G., Zaslavsky, O., \& Stein, M. K. (1990). Functions, graphs, and graphing: Tasks, learning, and teaching. Review of Educational Research, 60(1), 1-64.

Moore, K. C., \& Thompson, P. W. (2015). Shape thinking and students' graphing activity. In T. FukawaConnelly, N. E. Infante, K. Keene, \& M. Zandieh (Eds.), Proceedings of the 18th Meeting of the MAA Special Interest Group on Research in Undergraduate Mathematics Education (pp. 782-789). Pittsburgh, PA: RUME.

Oehrtman, M., Carlson, M., \& Thompson, P. W. (2008). Foundational reasoning abilities that promote coherence in students' function understanding. In M. P. Carlson \& C. Rasmussen (Eds.), Making the connection: Research and teaching in undergraduate mathematics education (pp. 27-42). Washington, DC: Mathematical Association of America.

Pierce, R., \& Stacey, K. (2004). Monitoring progress in algebra in a CAS active context: Symbol sense, algebraic insight and algebraic expectation. International Journal for Technology in Mathematics Education, 11(1), $3-11$.

Pierce, R., \& Stacey, K. (2007). Developing algebraic insight. Mathematics Teaching Incorporating Micromath, 203, 12-16.

Polya, G. (1945). How to solve it. Princeton, NJ: Princeton University Press.

Ruthven, K. (1990). The influence of graphic calculator use on translation from graphic to symbolic forms. Educational Studies in Mathematics, 21(5), 431-450.

Schwarz, B. B., \& Hershkowitz, R. (1999). Prototypes: Brakes or levers in learning the function concept? The role of computer tools. Journal for Research in Mathematics Education, 362-389.

Slavit, D. (1997). An alternate route to the reification of function. Educational Studies in Mathematics, 33(3), $259-281$.

Stylianou, D. A., \& Silver, E. A. (2004). The role of visual representations in advanced mathematical problem solving: An examination of expert-novice similarities and differences. Mathematical Thinking and Learning, 6(4), 353-387.

Swan, M. (2005). Standards Unit-Improving learning in mathematics: Challenges and strategies. Nottingham, UK: University of Nottingham. https://www.ncetm.org.uk/files/224/improving_learning_in_mathematicsi.pdf

Thompson, P. W. (2013). In the absence of meaning. In K. Leatham (Ed.), Vital directions for mathematics education research (pp. 57-93). New York, NY: Springer.

Tsamir, P., \& Bazzini, L. (2004). Consistencies and inconsistencies in students' solutions to algebraic 'single-value' inequalities. International Journal of Mathematical Education in Science and Technology, 35(6), 793-812.

Van Stiphout, I. M., Drijvers, P., \& Gravemeijer, K. (2013). The development of students' algebraic proficiency. International Electronic Journal of Mathematics Education, 8(2-3), 62-80.

Yerushalmy, M. (1997). Reaching the unreachable: Technology and the semantics of asymptotes. International Journal of Computers for Mathematical Learning, 2(1), 1-25.

Publisher's note Springer Nature remains neutral with regard to jurisdictional claims in published maps and institutional affiliations. 